\title{
Proteolytic degradation and potential role of onconeural protein cdr2 in neurodegeneration
}

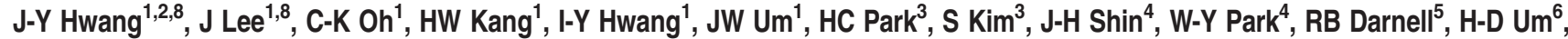 \\ $\mathrm{KC}$ Chung ${ }^{1}, \mathrm{~K} \mathrm{Kim}^{\star, 7}$ and YJ Oh,
}

Cerebellar degeneration-related protein 2 (cdr2) is expressed in the central nervous system, and its ectopic expression in tumor cells of patients with gynecological malignancies elicits immune responses by cdr2-specific autoantibodies and T lymphocytes, leading to neurological symptoms. However, little is known about the regulation and function of cdr2 in neurodegenerative diseases. Because we found that cdr2 is highly expressed in the midbrain, we investigated the role of cdr2 in experimental models of Parkinson's disease (PD). We found that cdr2 levels were significantly reduced after stereotaxic injection of 1-methyl-4phenylpyridinium (MPP $)$ into the striatum. cdr2 levels were also decreased in the brains of post-mortem PD patients. Using primary cultures of mesencephalic neurons and MN9D cells, we confirmed that MPP ${ }^{+}$reduces cdr2 in tyrosine hydroxylasepositive dopaminergic neuronal cells. The MPP ${ }^{+}$-induced decrease of cdr2 was primarily caused by calpain- and ubiquitin proteasome system-mediated degradation, and cotreatment with pharmacological inhibitors of these enzymes or overexpression of calcium-binding protein rendered cells less vulnerable to MPP'-mediated cytotoxicity. Consequently, overexpression of cdr2 rescued cells from $\mathrm{MPP}^{+}$-induced cytotoxicity, whereas knockdown of cdr2 accelerated toxicity. Collectively, our findings provide insights into the novel regulatory mechanism and potentially protective role of onconeural protein during dopaminergic neurodegeneration.

Cell Death and Disease (2016) 7, e2240; doi:10.1038/cddis.2016.151; published online 2 June 2016

Cerebellar degeneration-related protein 2 (cdr2), an onconeural protein, is associated with paraneoplastic cerebellar degeneration (PCD). ${ }^{1-3}$ Under physiological conditions, cdr2 expression is restricted to cerebellar Purkinje neurons, brain stem neurons, and testes. ${ }^{4,5}$ However, cdr2 is ectopically expressed in breast or ovarian tumors of PCD patients, resulting in the generation of autoantibodies ${ }^{6-8}$ that are associated with neurodegeneration of Purkinje neurons. ${ }^{9-12}$ Although the regulation of cdr2 is not well understood, an early study suggests that cdr2 is phosphorylated by PKN, ${ }^{13}$ and a more recent study shows that cdr2 is ubiquitinated by anaphase-promoting complex/cyclosome (APC/C) and degraded by proteasomes during the exit from mitosis. ${ }^{14}$ Despite these advances, the regulatory mechanisms and potential role of cdr2 in neurodegenerative disorders have not been explored.

Parkinson's disease (PD) is a neurodegenerative disorder characterized by a selective loss of dopaminergic neurons in the substantia nigra (SN) pars compacta that is associated with both motor defects and nonmotor symptoms. ${ }^{15}$ Mitochondrial dysfunction, oxidative stress, and inflammation are proposed to underlie the pathogenesis of familial and sporadic forms of $\mathrm{PD} .^{16,17}$ Accumulating evidence indicates that protease activation plays a critical role in the progression of neurodegeneration in PD. ${ }^{18-27}$ In our previous studies, we observed the activation of caspase and calpain in neurotoxininduced dopaminergic neurodegeneration ${ }^{28,29}$ and found that degradation of endogenous substrates by activated proteases leads to neurodegeneration. ${ }^{30,31}$ Therefore, in the present study, we investigated the expression and protease-mediated regulation of cdr2 in experimental models of PD. We found that cdr2 is downregulated by calpain and the ubiquitin proteasome system and that the restoration of cdr2 levels renders dopaminergic neurons less vulnerable to 1-methyl-4phenylpyridinium $\left(\mathrm{MPP}^{+}\right)$-mediated cytotoxicity. To our knowledge, it is the first report providing evidence that cdr2 is

\footnotetext{
${ }^{1}$ Department of Systems Biology, Yonsei University College of Life Science and Biotechnology, Seoul 120-749, Korea; ${ }^{2}$ Dominick P. Purpura Department of Neuroscience, Albert Einstein College of Medicine, New York, NY 10461, USA; ${ }^{3}$ Graduate School of Medicine, Korea University, Ansan 425-707, Gyeonggi-do, Korea; ${ }^{4}$ Division of Pharmacology, Department of Molecular Cell Biology, Sungkyunkwan University School of Medicine, Suwon 440-746, Gyeonggi-do, Korea; ${ }^{5}$ Laboratory of Molecular Neuro-Oncology, Howard Hughes Medical Institute, The Rockefeller University, New York, NY 10065, USA; ${ }^{6}$ Division of Radiation Cancer Biology, Korean Institute of Radiological \& Medical Sciences, Seoul 01812, Korea and ${ }^{7}$ Department of Brain and Cognitive Sciences, Daegu Gyeongbuk Institute of Science and Technology (DGIST), Daegu 711-873, Korea

*Corresponding author: K Kim, Department of Brain and Cognitive Sciences, DGIST, 333 Techno Jungang-daero, Hyeonoung-myeon, Dalseong-gun, Daegu 42988, Korea. Tel: +82 53785 6144; Fax: +82 53785 1219; E-mail: kyungjin@dgist.ac.kr

or YJ Oh, Department of Systems Biology, Yonsei University College of Life Science and Biotechnology, 134 Shinchon-Dong, Seodaemoon-Gu, Seoul 120-749, Korea. Tel: +82 22123 2662; Fax: +82 2312 5657; E-mail: yjoh@yonsei.ac.kr

${ }^{8}$ These two authors contributed equally to this work.

Abbreviations: PD, Parkinson's disease; cdr2, cerebellar degeneration-related protein 2; APC/C, anaphase-promoting complex/cyclosome; N2, N-2 supplement; MPP ${ }^{+}$, 1-methyl-4-phenylpyridinium; MTT, 3-(4,5-dimethylthiazol-2-yl)-2,5-diphenyltetrazolium bromide; PCD, paraneoplastic cerebellar degeneration; TH, tyrosine hydroxylase; GABA, $\gamma$-aminobutyric acid; Ub, ubiquitin; SN, substantia nigra; CSF, cerebrospinal fluid; Z-VAD-fmk, $N$-benzyloxycarbonyl-Val-Ala-Asp-fluoromethylketone; $\beta$-lactone, clasto-lactacystin $\beta$-lactone

Received 25.2.16; revised 21.4.16; accepted 05.5.16; Edited by A Verkhratsky
} 
a
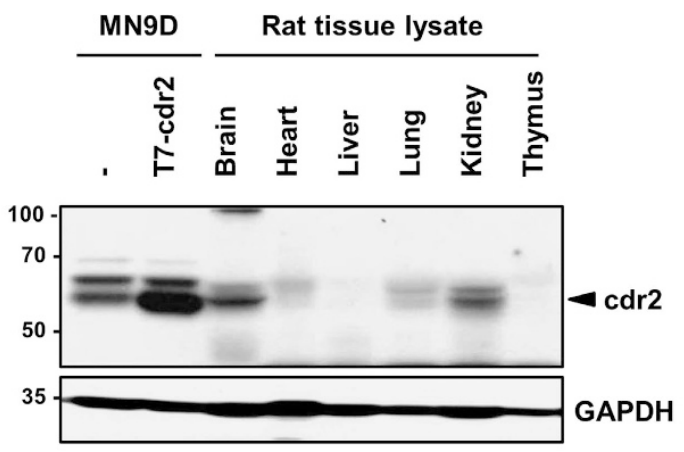

b

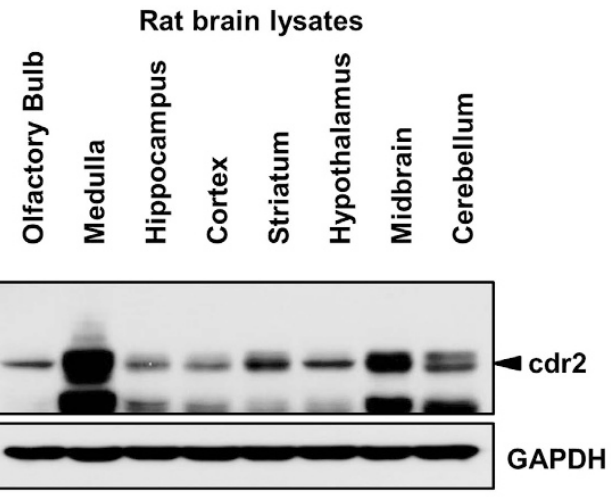

C

Rat brain cortical lysate
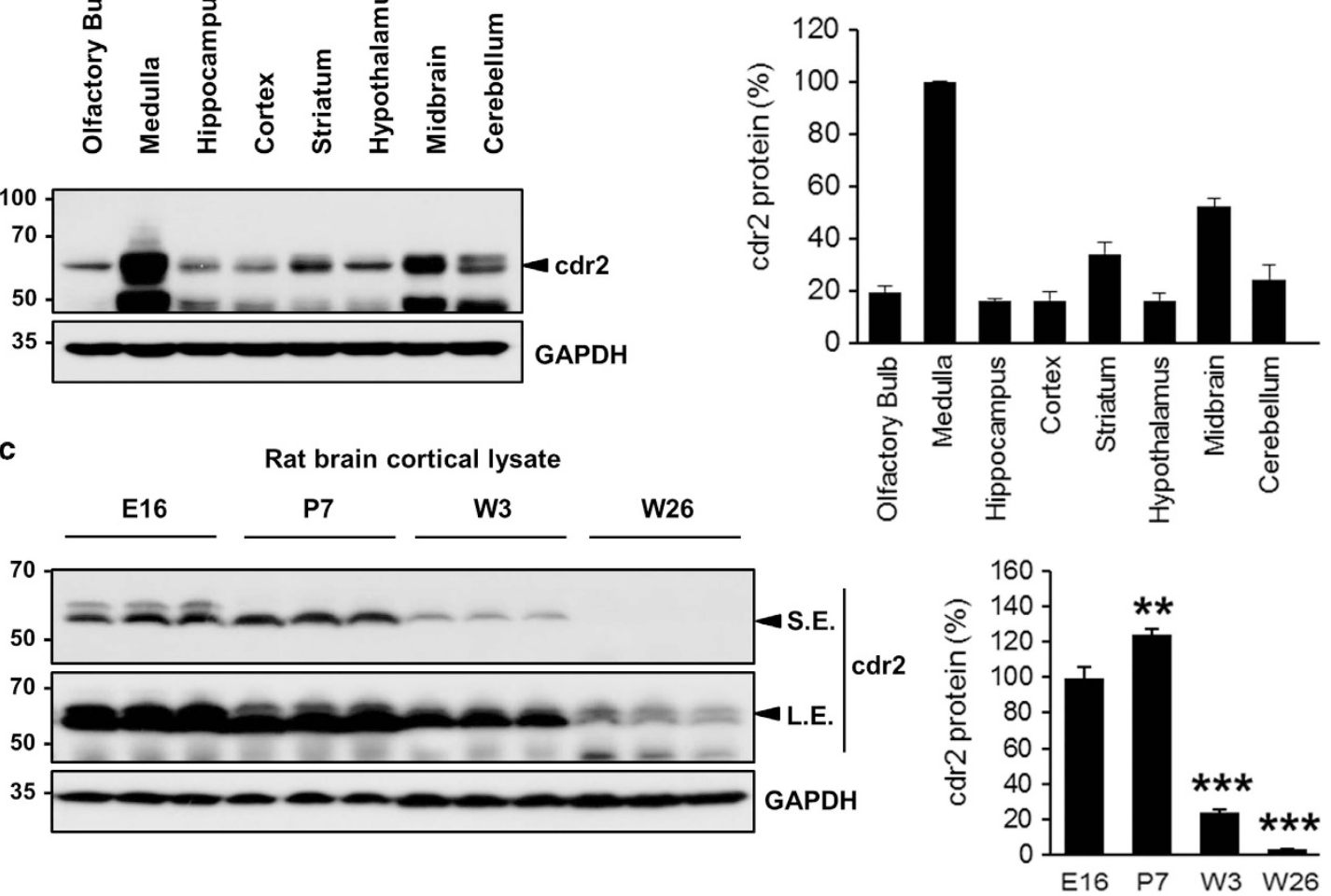

Figure 1 cdr2 expression in rat tissues. (a) cdr2 protein levels in tissue lysates $(50 \mu \mathrm{g})$ from adult rats ( 8 weeks old, male) were determined by immunoblotting with anti-cdr2 antibody. Lysates from MN9D dopaminergic neuronal cells transfected with or without T7-tagged mouse cdr2 were used as a positive control for the cdr2 band. Mouse monoclonal anti-GAPDH antibody was used as a loading control. (b) Immunoblot analysis comparing cdr2 levels across various brain regions. (c) Age-dependent expression of cdr2 in the cortex (E, embryonic day; P, postnatal day; W, postnatal week). Both short-term-exposed (S.E.) and long-term-exposed (L.E.) blots were presented. After normalization to GAPDH level, cdr2 levels in each lane were calculated relative to cdr2 levels in the medulla (b) or the E16 cortex (c). Data are shown as the mean \pm S.D. of three independent experiments. ${ }^{* \star} P<0.01 ;{ }^{* \star *} P<0.001$

proteolytically regulated and may play a neuroprotective role in drug-induced model of neurodegeneration.

\section{Results}

cdr2 is highly expressed in the midbrain of normal adult rats. Previous studies show that cdr2 is normally expressed in cerebellar Purkinje neurons but is ectopically expressed in breast and ovarian tumors of PCD patients. ${ }^{4,5,32}$ To further characterize the normal expression pattern of cdr2, lysates from various tissues from adult rats were immunoprobed with anti-cdr2 antibody. We found that cdr2 was highly expressed in the brain and kidney, whereas the heart and lung showed lower cdr2 expression (Figure 1a). This distinct spatial pattern of cdr2 expression prompted us to investigate cdr2 levels in more specific regions of the brain. We found that the medulla and midbrain showed the highest expression of cdr2, whereas the cerebellum, where Purkinje neurons reside, showed relatively lower cdr2 expression (Figure 1b). Double immunofluorescent localization of tyrosine hydroxylase (TH) and cdr2 revealed that both $\mathrm{TH}$-positive and -negative cells highly expressed cdr2 in the midbrain including ventral tegmental area, SN pars compacta, and SN pars reticulata (Supplementary Figure S1). Varying levels of cdr2 were expressed in other brain regions including hippocampus, 
cortex, striatum, and hypothalamus (Figure 1b). In a preliminary study, quite equivalent levels of cdr2 were detected in the spinal cord and olfactory bulb (data not shown). We also found abundant cdr2 expression in the cerebral cortex of prenatal and early postnatal rats and a dramatic downregulation in adult rats (Figure 1c), suggesting the temporal regulation of cdr2 expression in the brain. Invariably, we observed more than one band of cdr2. The in vitro phosphatase assay showed that the upper bands represent the phosphorylated forms of cdr2 (data not shown). Although we did not pursue this observation further, it is worthy to note that a serine/threonine kinase PKN (also known as protein kinase C-related kinase 1), which is involved in the pathogenesis of Alzheimer's disease and amyotrophic lateral sclerosis, ${ }^{33,34}$ interacts with and phosphorylates cdr2. ${ }^{13}$

cdr2 protein is decreased in the SN of rodent models of PD and post-mortem PD patients. PD is characterized by a selective loss of dopaminergic neurons in the SN and subsequent dopamine deprivation in the striatum. Our finding that cdr2 is highly expressed in the midbrain led us to examine the regulation of cdr2 in PD pathogenesis. First, we measured levels of cdr2 protein using rodent models of $\mathrm{PD}$ established by stereotaxic unilateral injection of $\mathrm{MPP}^{+}$ $(100 \mu \mathrm{mol})$ into the striatum. In the ipsilateral side of the SN pars compacta injected with $\mathrm{MPP}^{+}$for 2 weeks, the total number of TH-positive dopaminergic neurons was reduced by $\sim 29 \%$ over the sham control group or the contralateral side of $\mathrm{MPP}^{+}$injection, as determined by unbiased stereological cell counts (Supplementary Figure S2a). At 1 or 2 weeks after $\mathrm{MPP}^{+}$injection into the striatum, cdr2 levels were reduced in the ipsilateral side of the SN pars compacta (Figure 2a and Supplementary Figure S2b). No discernible decrease of cdr2 was detected in the contralateral side of the midbrain or in sham controls. Any obvious decrease of cdr2 was not observed in the SN pars reticulata (Supplementary Figure S2b). Similar pattern of cdr2 reduction in the ipsilateral side of the midbrain was also observed in rats that received stereotaxic injection of $\mathrm{MPP}^{+}$into the middle forebrain bundle, another rodent model of PD (data not shown). This $\mathrm{MPP}^{+}$-induced decrease in cdr2 levels was associated with a reduction of $\mathrm{TH}$ protein, indicating that decrease in cdr2 levels may be ascribed to a loss of $\mathrm{TH}$-positive dopaminergic neurons or lower expression levels of cdr2 in dying dopaminergic neurons or both. Therefore, we next determined whether cdr2 levels are decreased in individual TH-positive dying neurons in the SN. At 2 weeks after $\mathrm{MPP}^{+}$injection, cdr2 levels were well preserved in the cytosol of TH-positive neurons in sham controls or the contralateral side of injection (Figure 2b). However, TH-positive neurons in the ipsilateral side showed morphology typical of retracted neurites, and their cdr2 levels were markedly decreased (Figure 2b, arrows). Quantitative analysis of fluorescence intensity showed a significant decrease in cdr2 levels in ipsilateral individual $\mathrm{TH}$-positive neurons (Figure $2 \mathrm{~b}$, right). When we measured cdr2 levels in the SN of post-mortem PD patients and age-matched controls (Figure 3a), we interestingly observed a significant decrease in cdr2 levels in all four PD brains, whereas levels of HSP70, a molecular chaperone, were not altered (Figure 3b).
$\mathrm{MPP}^{+}$-induced reduction of cdr2 occurs in the TH-positive dopaminergic neurons. To further determine whether the $\mathrm{MPP}^{+}$-induced decrease of cdr2 occurs in $\mathrm{TH}$-positive dying dopaminergic cells, we used primary cultures of dopaminergic neurons derived from the rat embryonic mesencephalon or the MN9D dopaminergic neuronal cell line. Exposure of primary cultures of mesencephalic neurons to $3 \mu \mathrm{M} \mathrm{MPP}{ }^{+}$for $36 \mathrm{~h}$ induced morphological changes such as neurite retraction and fragmentation (Figure 4a). In this condition, quantitative analysis indicated that cdr2 levels were significantly reduced in $\mathrm{TH}$-positive dying neurons following $\mathrm{MPP}^{+}$treatment (Figure 4a, right panel). In contrast, there were no discernible changes of cdr2 levels in $\gamma$-aminobutyric acid (GABA)-positive neurons in the same culture. These data along with our finding that cdr2 is preserved in neurons of $\mathrm{SN}$ pars reticulata suggest that the $\mathrm{MPP}^{+}$-induced decrease in cdr2 levels is cell-type specific. The MN9D cell line, a fusion product of mesencephalic dopaminergic neurons and N18TG neuroblastoma, was previously demonstrated to synthesize, release, and take up dopamine. ${ }^{35,36}$ As determined by immunocytochemistry, exposure of MN9D dopaminergic cells to $50 \mu \mathrm{M} \mathrm{MPP}^{+}$for $36 \mathrm{~h}$ or longer caused a significant reduction in cdr2 levels, whereas numbers of cells in culture remained the same regardless of drug treatment (Figure 4b). Immunoblot analysis indicated that $\mathrm{MPP}^{+}$causes a time-dependent reduction of cdr2 (Figure 4c), Taken together, our data indicated that decrease in cdr2 occurs in dying $\mathrm{TH}$-positive neurons and is not simply due to $\mathrm{MPP}^{+}$-induced loss of dopaminergic neurons.

Activated calpain and the ubiquitin proteasome system are responsible for $\mathrm{MPP}^{+}$-induced decrease of cdr2. Having established that cdr2 is downregulated in the brains of experimental models of PD as well as post-mortem PD patients, we next investigated the mechanism underlying $\mathrm{MPP}^{+}$-induced reduction of cdr2. $\mathrm{MPP}^{+}$-induced cell death is accompanied by or results from a burst of intracellular free $\mathrm{Ca}^{2+}$, leading to activation of calpain, a $\mathrm{Ca}^{2+}$-dependent cysteine protease in MN9D cells as previously demonstrated by us. $^{30,37,38}$ Therefore, we determined whether $\mathrm{Ca}^{2+}$-mediated calpain activation is responsible for $\mathrm{MPP}^{+}$-induced reduction of cdr2. As shown in Figure 5a, many MN9D cells stained positive for fluo-3, a $\mathrm{Ca}^{2+}$-sensitive fluorescent dye, $36 \mathrm{~h}$ after $50 \mu \mathrm{M}$ $\mathrm{MPP}^{+}$treatment. Quantitative analysis revealed an approximately fourfold increase in the intensity of fluo-3-stained MN9D cells relative to untreated control cells $36 \mathrm{~h}$ after $\mathrm{MPP}^{+}$treatment (data not shown). Moreover, fodrin, a general calpain substrate, was cleaved in a time-dependent manner (Figure 5b), indicating that a rise of intracellular free $\mathrm{Ca}^{2+}$ leads to calpain activation in $\mathrm{MPP}^{+}$-treated MN9D cells. We next specifically inquired whether cdr2 is a substrate of calpain by incubating lysates of MN9D cells in calpain-activating conditions. We found that the addition of either $\mathrm{m}$ - or $\mu$-calpain caused complete degradation of endogenous cdr2 (Figure 5c), and this event was blocked in the presence of calpeptin, a cell-permeable calpain inhibitor. To demonstrate that cdr2 is a direct substrate of activated calpain, we performed a calpain cleavage assay using in vitro translated $\left[{ }^{35} \mathrm{~S}\right]$-labeled cdr2. Both $\mathrm{m}$ - and $\mu$-calpain led to complete degradation of cdr2 (Figure $5 d$ ) that was inhibited in the presence of calpeptin or MG132, another calpain inhibitor. ${ }^{39}$ 
Rat STR stereotaxic injection

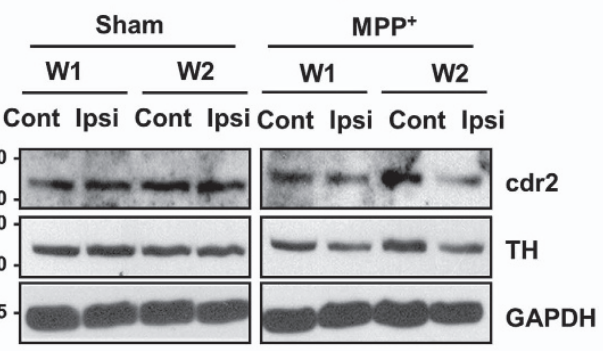

b

Rat STR stereotaxic injection
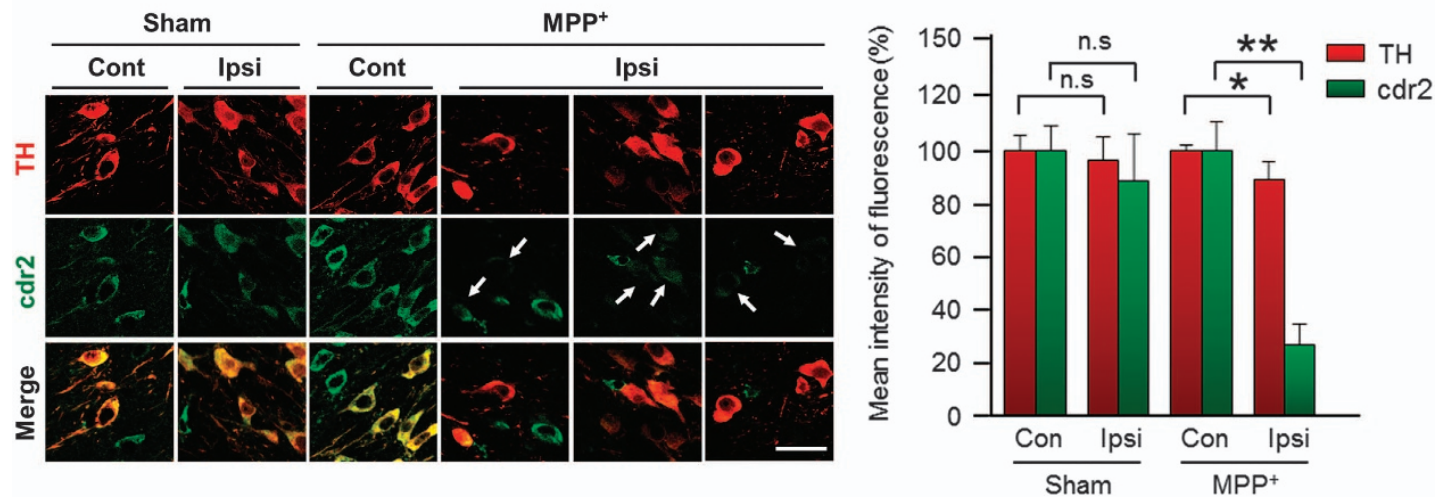

Figure 2 Downregulation of cdr2 in MPP+-injected rat brains. (a) Rats received a unilateral stereotaxic injection of saline (sham) or MPP (100 $\mu$ mol) into the striatum (STR). The indicated periods (W, week) after surgery, and cdr2 levels in the ipsilateral (Ipsi) or contralateral (Cont) SN were determined by immunoblot analysis using anti-cdr2 antibody. Mouse monoclonal anti-TH antibody was used to assess the severity of damage to dopaminergic neurons. (b) At 2 weeks after stereotaxic injection of MPP into the STR, SN sections were immunolabeled with anti-TH antibody and human PCD patient CSF followed by incubation with fluorescence-tagged secondary antibodies. Arrows indicate cdr2 staining in TH-positive neurons after MPP+ treatment. Scale bar: $40 \mu \mathrm{m}$. The mean intensity of TH and cdr2 fluorescence in the Ipsi side was measured and expressed as a percentage of that in the sham-treated Cont side. Data were obtained from five randomly selected microscopic fields per section, with 16-20 sections per rat ( 8-10 neurons per section). Data are shown as the mean \pm S.D. of $4-5$ rats. ${ }^{*} P<0.05 ;{ }^{* *} P<0.01$; n.s., not significant

We next sought to investigate whether other proteases are involved in $\mathrm{MPP}^{+}$-induced degradation of cdr2. MN9D cells were treated with $50 \mu \mathrm{M} \mathrm{MPP}^{+}$in the presence or absence of various protease inhibitors. Again, $\mathrm{MPP}^{+}$-induced degradation of cdr2 was significantly inhibited in the presence of calpeptin or MG132 (Figure 6a). Because MG132 can inhibit different types of proteases, including calpain, serine proteases, and proteasomes, we used a more specific irreversible proteasome inhibitor, clasto-lactacystin $\beta$-lactone. Cotreatment of MN9D cells with clasto-lactacystin $\beta$-lactone significantly blocked $\mathrm{MPP}^{+}$-induced degradation of cdr2, whereas cotreatment with z-VAD, a pan-caspase inhibitor, had no effect (Figure 6a). To directly determine whether cdr2 is also a substrate for the ubiquitin proteasome system, we performed in vitro ubiquitination assay using $\left[{ }^{35} \mathrm{~S}\right]$-labeled cdr2 protein. We found that poly-ubiquitinated bands of cdr2 appeared in a reaction mixture containing $E 1, E 2$, and $E 3$ enzymes (Figure 6b). To test whether $\mathrm{MPP}^{+}$induces the ubiquitination of cdr2, MN9D cells transiently transfected with T7-tagged cdr2 and HA-tagged ubiquitin (HA-Ub) were exposed to $50 \mu \mathrm{M}$ $\mathrm{MPP}^{+}$. Immunoprecipitation and immunoblot assay revealed that the poly-ubiquitinated cdr2 first emerged at $24 \mathrm{~h}$ and further increased $48 \mathrm{~h}$ after $\mathrm{MPP}^{+}$exposure, whereas no discernible ubiquitination bands were found in untreated control cells (Figure 6c). Consistently, endogenous cdr2 was also ubiquitinated in MN9D cells transiently transfected with $\mathrm{HA}-\mathrm{Ub}$ after exposure to $50 \mu \mathrm{M} \mathrm{MPP}^{+}$for $36 \mathrm{~h}$ (Figure $6 \mathrm{~d}$ ). Taken together, our results indicate that $\mathrm{MPP}^{+}$-induced degradation of cdr2 is mediated by at least two independent mechanisms: calpain and the ubiquitin proteasome system.

cdr2 protects dopaminergic cells from $\mathrm{MPP}^{+}$-induced cell death. Our observation of cdr2 downregulation in PD brain models and post-mortem human PD brains prompted us to investigate a functional role of cdr2 during dopaminergic neurodegeneration. Based on our observation that cdr2 is degraded by activated calpain following $\mathrm{MPP}^{+}$treatment, we first asked whether chelation of cytosolic $\mathrm{Ca}^{2+}$ and/or inhibition of calpain could rescue cells from $\mathrm{MPP}^{+}$-mediated cytotoxicity. To test this hypothesis, we first utilized MN9D cells stably expressing calbindin-D-28K with four conserved $\mathrm{EF}$ hand domains and $\mathrm{Ca}^{2+}$ buffering activity. ${ }^{40,41}$ We found that $\mathrm{MPP}^{+}(50 \mu \mathrm{M})$-induced decrease of cdr2 and appearance of calpain-cleaved fodrin were attenuated in all three independent MN9D/calbindin-D-28K cell lines (\#1, \#2, and \#3) compared with a mock-transfected control cell line (MN9D/ $\mathrm{Neo}$; Figure 7a). Consequently, all three calbindin-expressing cell lines showed less vulnerability to $50 \mu \mathrm{M} \mathrm{MPP}^{+}$treatment as determined by MTT (3-(4,5-dimethylthiazol-2-yl)-2,5diphenyltetrazolium bromide) reduction assay (Figure $7 \mathrm{~b}$ ). 
a

\begin{tabular}{|c|c|c|c|c|c|}
\hline & Final Diagnosis & Age & Sex & Race & PMD \\
\hline \multirow{4}{*}{ Control } & Control & 87 & $\mathbf{F}$ & w & 7 \\
\hline & Control & 89 & M & w & 8.5 \\
\hline & Control & 71 & M & w & 16 \\
\hline & Control & 79 & M & w & 16 \\
\hline \multirow{4}{*}{ PD } & PD w/dementia & 76 & M & w & 17 \\
\hline & $\begin{array}{l}\text { PD w/dementia, } \\
\text { neurodegeneration, } \\
\text { occipital infarct }\end{array}$ & 83 & M & w & 5 \\
\hline & PD, Neocortical & 71 & M & w & 8 \\
\hline & PD w/dementia & 73 & M & w & 6.5 \\
\hline
\end{tabular}

b Human postmortem SN
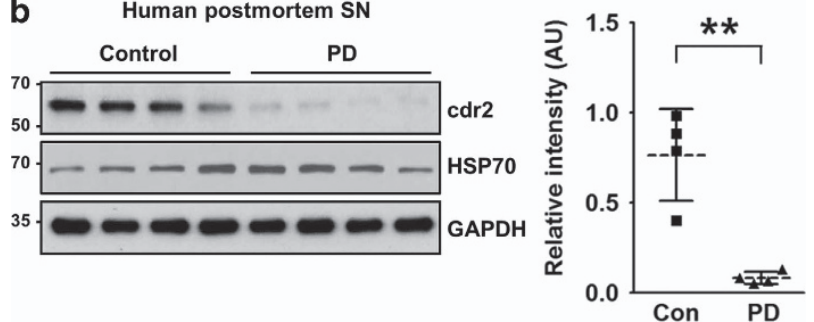

Figure 3 Downregulation of cdr2 in brains from post-mortem PD patients. (a) Characteristics of PD patients and age-matched controls, including diagnosis, age, sex, race, and post-mortem delay (PMD; h). (b) Tissue lysates from the SN of post-mortem humans were subjected to immunoblot analysis using anti-cdr2 antibody and anti-HSP70 antibody. After normalization against GAPDH, relative intensity of cdr2 in brains from post-mortem PD patients or age-matched controls. Data are shown as mean \pm S.D. ${ }^{* \star} P<0.01$

Similarly, pharmacological inhibition of calpain by calpeptin or MG132, which partially preserved cdr2 levels, attenuated $\mathrm{MPP}^{+}$-induced MN9D cell death (Figures 7c-f), suggesting that the reduced vulnerability of calbindin-expressing cells or calpeptin/MG132-treated MN9D cells may be in part attributed to preserved cdr2 levels. To directly examine whether the level of cdr2 itself affects cell survival upon exposure to $50 \mu \mathrm{M} \mathrm{MPP}^{+}$, we established three MN9D cell lines stably overexpressing T7-tagged cdr2 and two MN9D cell lines in which cdr2 was stably silenced by transfection with short hairpin RNA (shRNA; Figures $8 a$ and c). MTT reduction assay using two highly cdr2-expressing cell lines (MN9D/cdr2 \#2 and \#3) indicated that overexpression of cdr2 protects cells from $\mathrm{MPP}^{+}$-induced cytotoxicity (Figure 8b). In contrast, $\mathrm{MPP}^{+}$-induced cell death was accelerated in cdr2-silenced MN9D cells (Figure 8d). Quite similar pattern was observed when stable MN9D cells were exposed to $50 \mu \mathrm{M} \mathrm{MPP}^{+}$for varying time periods (Supplementary Figure S3). Interestingly, we observed that cdr2 is also decreased during 6-hydroxydopamine (6-OHDA)-induced neuronal death (Supplementary Figures $\mathrm{S} 4 \mathrm{a}$ and b). As we previously demonstrated, ${ }^{28,29} 6$-OHDA led to reactive oxygen speciesdependent MN9D cell death. As a consequence, 6-OHDAmediated decrease in cdr2 was largely inhibited in the presence of $\mathrm{N}$-acetyl-L-cysteine but not calpeptin. These data suggest that an additional calpain-independent degradation pathway may be involved in 6-OHDA-mediated decrease of cdr2. We also observed that 6-OHDA-induced cell death was significantly blocked in cdr2-overexpressing MN9D cells
(Supplementary Figure S4c). Collectively, our results suggest that cdr2 may play a neuroprotective role in drug-induced dopaminergic neurodegeneration.

\section{Discussion}

Although cdr2 mRNA is widely expressed, cdr2 protein expression is restricted to immune-privileged sites including the testis, brain stem, and cerebellum, suggesting that cdr2 expression is regulated by a post-transcriptional mechanism. ${ }^{4,5}$ Here, we examined cdr2 expression patterns in several brain regions and found that the second most prominent site of expression was the midbrain. We also observed high levels of cdr2 expression in the striatum, a target of dopaminergic projections from the SN pars compacta. Therefore, we investigated whether cdr2 levels are altered in dopaminergic neurodegeneration. Indeed, we found lower cdr2 protein levels in the brains of post-mortem PD patients and animal PD models established by stereotaxic injection of $\mathrm{MPP}^{+}$into the striatum. Using both cultured MN9D dopaminergic cells and primary cultures of mesencephalic neurons challenged with $\mathrm{MPP}^{+}$, we found decreased levels of cdr2 in $\mathrm{TH}$-positive dying dopaminergic neurons but not in GABAergic neurons. This cell-type specificity can be addressed by the fact that GABAergic neurons is more resistant to $\mathrm{MPP}^{+}$or rotenone, another PD-related drug targeting mitochondria complex $\mathrm{I}^{42}$

We showed that cdr2 degradation was mediated primarily by calpain and the ubiquitin proteasome system. Consequently, pharmacological inhibition of these enzymes successfully blocked $\mathrm{MPP}^{+}$-induced degradation of cdr2 and subsequent dopaminergic neurodegeneration. Previous studies, including ones from our laboratory, report that $\mathrm{MPP}^{+}$induced calpain activation via increased intracellular $\mathrm{Ca}^{2+}$ leads to cleavage of numerous cellular substrates and contributes to neuronal cell death, ${ }^{28,30,43,44}$ supporting the notion that degradation of critical cellular proteins by activated calpain may be linked to dopaminergic neurodegeneration. An increase in intracellular free $\mathrm{Ca}^{2+}$ is also known to occur in acute neurodegenerative conditions such as ischemic stroke and spinal cord injury. ${ }^{45-48}$ In preliminary studies, we found that cdr2 levels were decreased in a rodent ischemic stroke model established by middle cerebral artery occlusion and a contusion spinal cord injury model (data not shown), suggesting that cdr2 may be degraded by activated calpain during both acute and chronic neurodegeneration. Previously, it has been demonstrated that cdr2 undergoes APC/Cmediated poly-ubiquitination during the exit from mitosis. ${ }^{14}$ Although we did not determine the relationship between $\mathrm{MPP}^{+}$-induced ubiquitination of $\mathrm{cdr} 2$ and $\mathrm{APC} / \mathrm{C}$ activity in MN9D cells, the results of our in vitro and cell-based ubiquitination assays raise the possibility that cdr2 is polyubiquitinated and subject to proteasome-mediated degradation after $\mathrm{MPP}^{+}$treatment. In a separate study, we found that both Parkin and SIAH bind to cdr2 in HEK293 cells and N2 (N-2 supplement) neuroblastoma (data not shown). However, we do not yet have clear evidence of cdr2 poly-ubiquitination by either of these two E3 ligases. Therefore, further attempts are necessary to identify the specific E3 ligase for cdr2 ubiquitination. 
a

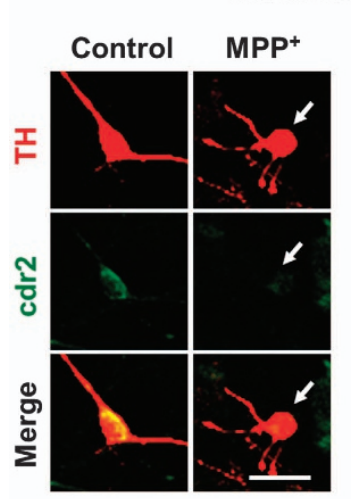

Rat midbrain culture

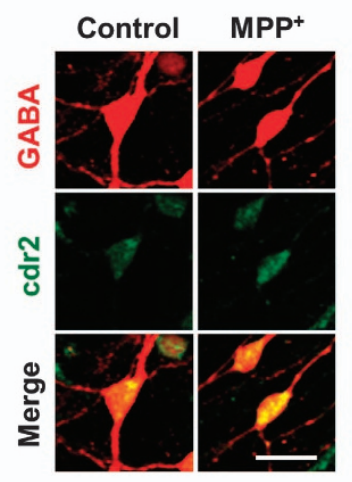

b

MN9D cells

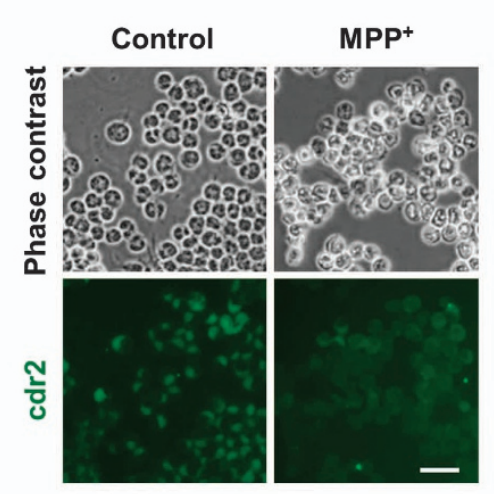

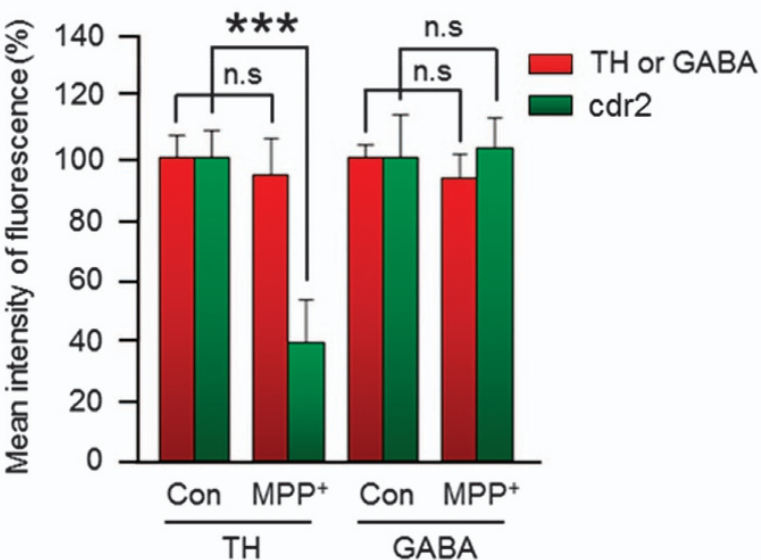

C
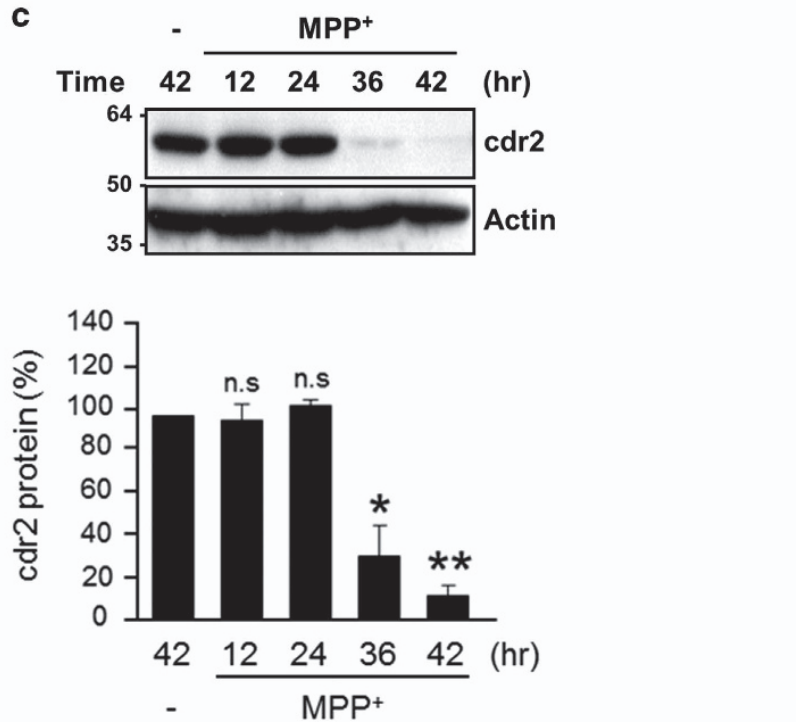

Figure 4 Downregulation of cdr2 in MPP+-treated dopaminergic neurons. (a) Primary cultures of rat mesencephalic dopaminergic neurons at DIV 5 or 6 were treated with or without $3 \mu \mathrm{M} \mathrm{MPP}^{+}$for $36 \mathrm{~h}$. Cells were subjected to double immunofluorescent staining using human PCD patient CSF and anti-TH antibody or anti-GABA antibody. TH-positive neurons having retracted or fragmented neurites are indicated by white arrows. Scale bars: $20 \mu \mathrm{m}$. Mean intensity of TH/GABA or cdr2 fluorescence was measured and expressed as a percentage of that in sham-treated control cultures. Data were obtained from 300-400 neurons from 5 to 10 randomly selected microscopic fields. Data are shown as the mean \pm S.D. of three independent experiments. ${ }^{* * *} \mathrm{P}<0.001$; n.S., not significant. (b) MN9D dopaminergic neuronal cells exposed to $50 \mu \mathrm{M}$ MPP ${ }^{+}$for $36 \mathrm{~h}$ were immunostained using human PCD patient CSF. Phase-contrast and fluorescent images were taken using an Axiovert 100. Scale bar: $50 \mu \mathrm{m}$. (c) Lysates (50 $\mu \mathrm{g}$ ) from MN9D cells exposed to $50 \mu \mathrm{M} \mathrm{MPP}^{+}$for the indicated time period were subjected to immunoblot analysis using anti-cdr2 antibody. Anti-actin antibody was used as a loading control. After normalization against actin, levels of cdr2 were expressed as a percentage of that in untreated control cells. Data are shown as the mean \pm S.D. of three independent experiments. ${ }^{*} P<0.05 ;{ }^{* *} P<0.01$; n.s., not significant

Our previous study showed that calpain-mediated cleavage of optineurin, peripherin, or arsenical pump-driving ATPase occurs in $\mathrm{MPP}^{+}$-treated MN9D dopaminergic cells, and any treatments that restore their protein levels or overexpression of one of these substrates rescue MN9D cells from MPP ${ }^{+}$ mediated cytotoxicity. ${ }^{30}$ In accordance with the results of pharmacological inhibition of calpain, MN9D cells overexpressing calbindin-D-28K showed preservation of cdr2, resulting in more resistance to $\mathrm{MPP}^{+}$-induced cytotoxicity. Similarly, we demonstrated that MN9D cells overexpressing cdr2 are less vulnerable to $\mathrm{MPP}^{+}$-induced cytotoxic damage. Conversely, $\mathrm{MPP}^{+}$-induced cell death was accelerated in MN9D cells subjected to shRNA-mediated silencing of cdr2, suggesting that cdr2 protein levels may be positively correlated with the rate of neuroprotection in MN9D cells after MPP ${ }^{+}$treatment.
Therefore, we are tempting to suggest that cdr2 exerts a certain neuroprotective function and thus its degradation may be associated with drug-induced neurodegeneration. Intriguingly, we also found that shRNA-mediated knockdown of cdr2 in cultured hippocampal neurons enhanced spontaneous apoptotic cell death, raising the possibility that cdr2 may have a neuroprotective role during early development (Supplementary Figure S5). Furthermore, cdr2 morpholinoinjected zebrafish embryos showed massive death of neural progenitor cells and post-mitotic differentiated neuronal cells in the spinal cord (Supplementary Figure S6). Considering that developing neurons undergo programmed cell death to control the number of neural progenitor cells and optimize neural connections between differentiating neurons and their targets in both vertebrates and invertebrates, ${ }^{49}$ our 
a
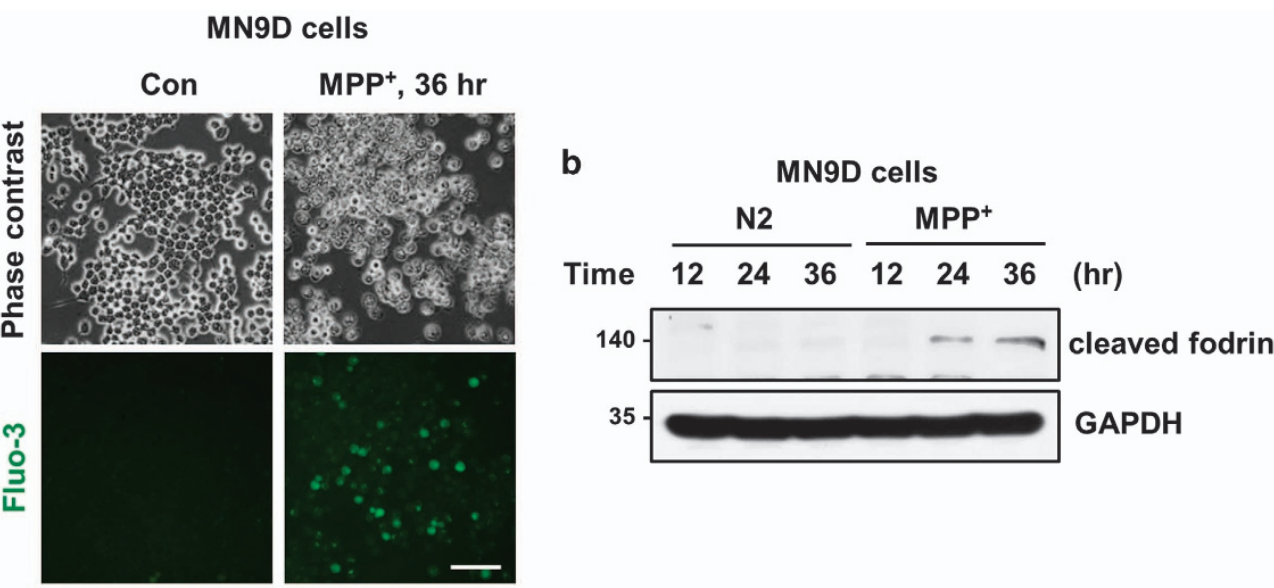

C Cell-based cleavage assay

d

In vitro cleavage assay

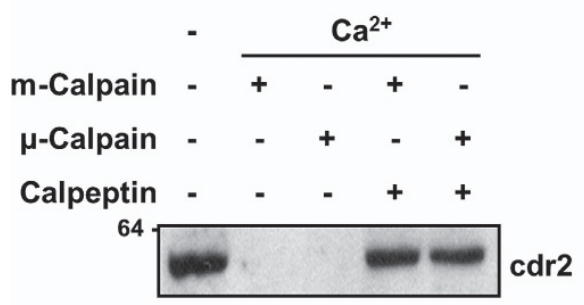

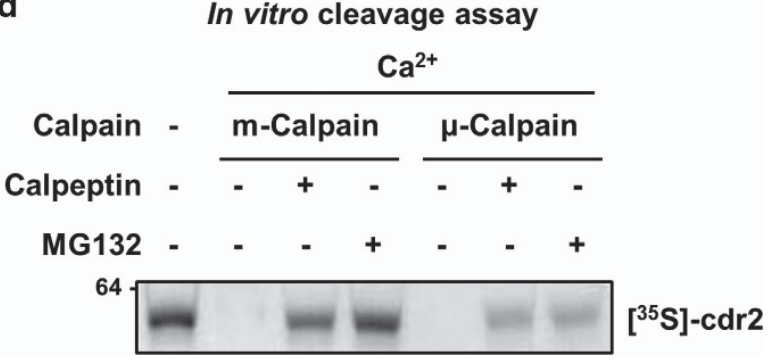

Figure 5 Degradation of cdr2 by calpain. (a) MN9D cells were treated with $50 \mu \mathrm{M} \mathrm{MPP}{ }^{+}$for $36 \mathrm{~h}$. Cells were then loaded with Fluo-3/AM dye and examined under a fluorescence microscope to detect levels of intracellular free $\mathrm{Ca}^{2+}$. Scale bar: $100 \mu \mathrm{m}$. (b) Lysates (50 $\left.\mu \mathrm{g}\right)$ from MN9D cells treated with or without $50 \mu \mathrm{M}$ MPP for the indicated time period were subject to immunoblot analysis using monoclonal anti-fodrin antibody recognizing calpain-cleaved band. (c) Lysates from MN9D cells $(50 \mu \mathrm{g})$ were incubated with recombinant m-calpain ( 0.343 units) or $\mu$-calpain $\left(0.134\right.$ units) in the presence of $1 \mathrm{mM} \mathrm{CaCl}_{2}$. Calpeptin $(50 \mu \mathrm{M})$ was added to the lysates to block calpain activity. After the reaction, immunoblot analysis using anti-cdr2 antibody was performed to detect remaining cdr2. (d) For in vitro cleavage assay, [ ${ }^{35} \mathrm{~S}$-labeled cdr2 was incubated with $\mathrm{m}$-calpain ( 0.343 units) or $\mu$-calpain ( 0.134 units) in the presence of $1 \mathrm{mM} \mathrm{CaCl}$. Calpeptin $(50 \mu \mathrm{M})$ or $\mathrm{MG} 132(10 \mu \mathrm{M})$ was added to the reaction mixtures. After incubation, all samples were separated by SDS-PAGE and subjected to autoradiography

preliminary findings suggest that cdr2 may be a key regulator and not merely a bystander of neuronal cell survival, although it is highly speculative at present.

Previous studies by others indicate that cdr2 is primarily present in the cytoplasm and has a leucine zipper motif. ${ }^{6,13,50,51}$ Therefore, cdr2 could bind to other proteins with a leucine zipper motif and exert transcriptional transactivation activity via binding to DNA. For example, cdr2 captures c-myc in the cytoplasm that prevents the transactivation of pro-apoptotic genes by nuclear c-myc activity in cerebellar Purkinje neurons, ${ }^{10}$ indicating that cdr2 can block c-mycmediated apoptosis. As another example, cdr2 interacts with a nuclear helix-loop-helix leucine zipper protein, MRG $X,{ }^{52}$ and coexpression of cdr2 and MRG X prevents MRG X-induced glioblastoma cell death. The same laboratory also reports that cdr2 binds to the cell cycle-related protein MRG15 and that overexpression of cdr2 inhibits the derepression of B-myb transcriptional activity by MRG15. ${ }^{53}$ The B-myb transcription factor is induced in response to apoptotic stimuli, and its knockdown in neurons is protective against nerve growth factor deprivation or drug-induced cell death accompanying DNA damage. ${ }^{54}$ Therefore, cdr2 may serve a neuroprotective role by repressing B-myb promoter activity. Although we did not attempt to determine whether these scenarios hold true in MN9D cells, we observed that $\mathrm{MPP}^{+}$treatment decreased levels of c-myc in the nucleus regardless of whether cells overexpressed cdr2 (data not shown). Therefore, it seems that a neuroprotective role of cdr2 cannot be ascribed to its known regulation of the c-myc-mediated cell death pathway, at least in MN9D cells. We also found that cor2 has a chromosome segregation ATPase domain as determined by a database search and can bind to ATP as determined by in vitro ATP binding assay (data not shown). Similarly, a colocalization study performed in our laboratory indicates that cdr2 may bind to microtubules in MN9D cells (data not shown). At present, we do not know whether and how these activities of cdr2 are related to its neuroprotective role in neuronal cells. Therefore, further studies delineating the biological and neuroprotective activity of cdr2 in the nervous system are required.

In summary, we characterized the cdr2 expression profile in various regions of the brain, and examined its regulation by activated proteases and its neuroprotective function during neurotoxin-induced dopaminergic neurodegeneration. Our findings indicate that the negative regulation of cor2 levels by activated calpain and the ubiquitin proteasome system may contribute to $\mathrm{MPP}^{+}$-induced neuronal cell death. Unveiling the detail mechanisms involved in regulation of cdr2 would expand our understanding of the potential role of cdr2 in pathological neurodegeneration. 
Materials and Methods

Animals, stereotaxic surgery, and post-mortem human brains. All experimental animal procedures were in accordance with the National Institutes of Health Guide for the Care and Use of Laboratory Animals and approved by the Institutional Animal Care and Use Committees of Yonsei University. To measure tissue-specific expression of cdr2 protein, various body parts including subregions

a
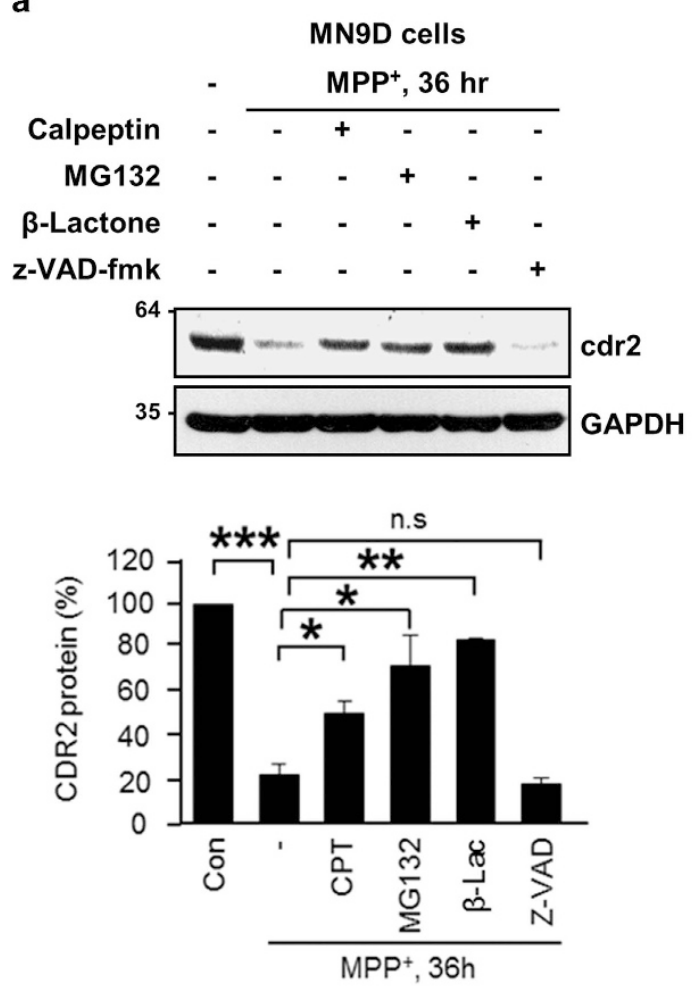

C

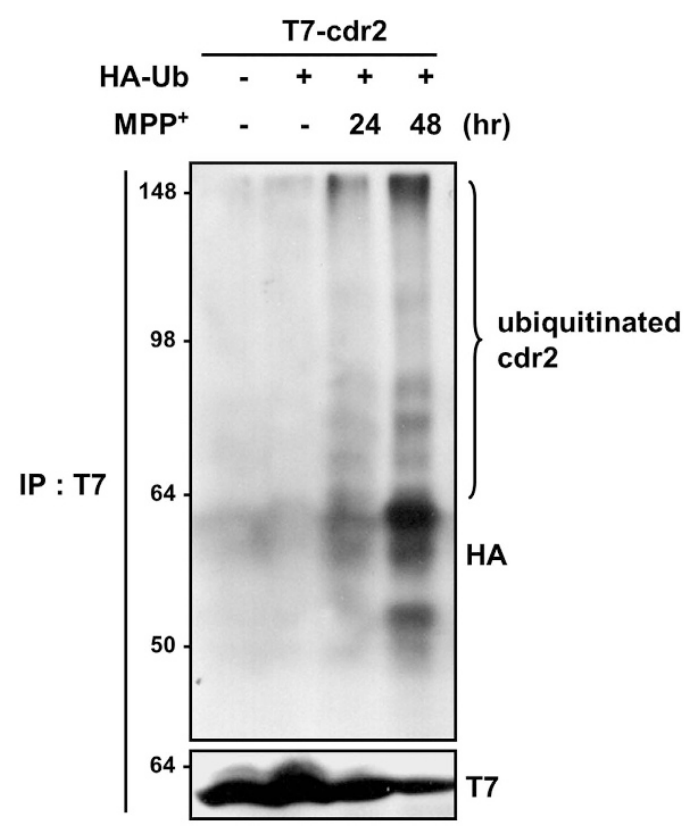

of the brain were surgically removed from Sprague-Dawley (SD) rats (Orientbio, Seongnam, Korea) at different ages. For other groups of rats, stereotaxic surgery was performed as previously described with modifications. ${ }^{55}$ Briefly, female SD rats (250-280 g; Daehan Biolink, Deajon, Korea) were anesthetized using an intraperitoneal injection of chloral hydrate $(360 \mathrm{mg} / \mathrm{kg})$ and placed in a stereotaxic apparatus (Kopf Instrument, Tujunga, CA, USA). Each rat received a unilateral

b In vitro ubiquitination assay
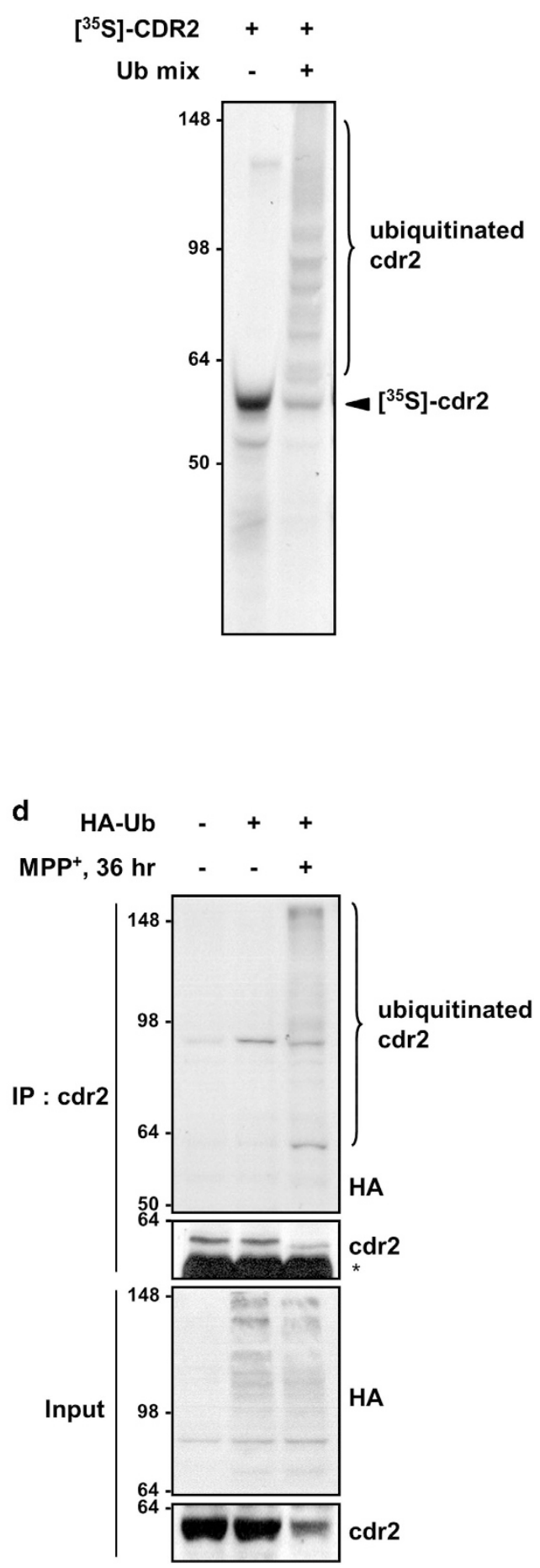
injection of $\mathrm{MPP}^{+}$(Sigma, St. Louis, MO, USA) or sterilized saline into the right striatum $(100 \mathrm{nmol} ;+1 \mathrm{~mm}$ anteroposterior, $-2.5 \mathrm{~mm}$ mediolateral, and $-4.5 \mathrm{~mm}$ dorsoventral relative to bregma) according to Paxinos and Watson (1998). ${ }^{56}$
Injections were performed using a Hamilton syringe equipped with a 26S-gauge beveled needle driven by a syringe pump (K.D. Scientific, Holliston, MA, USA). The needle was slowly retracted $10 \mathrm{~min}$ after the injection. On the predetermined day a

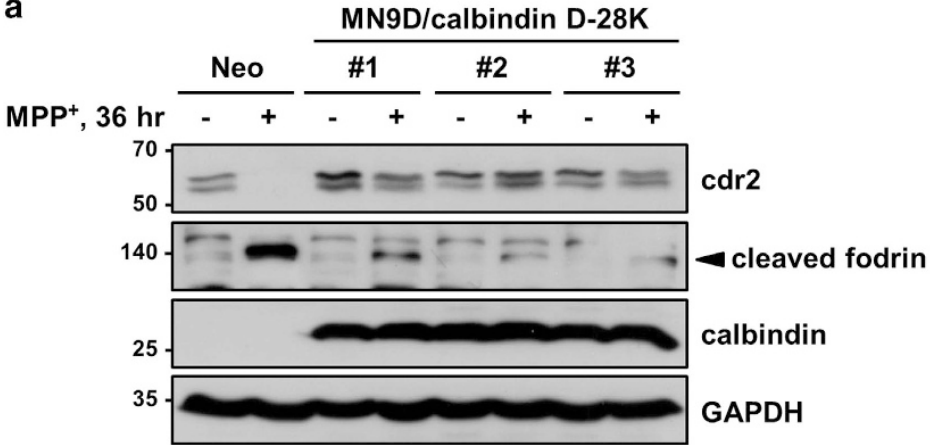

C

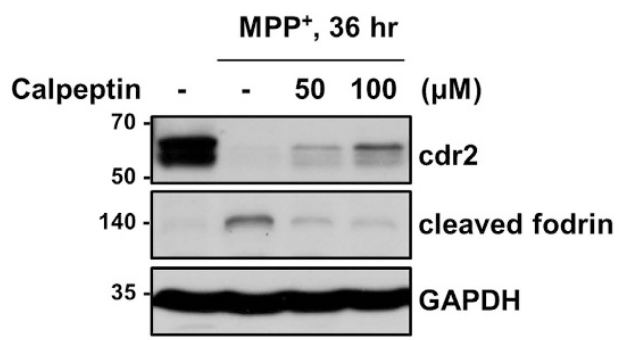

e

MN9D cells

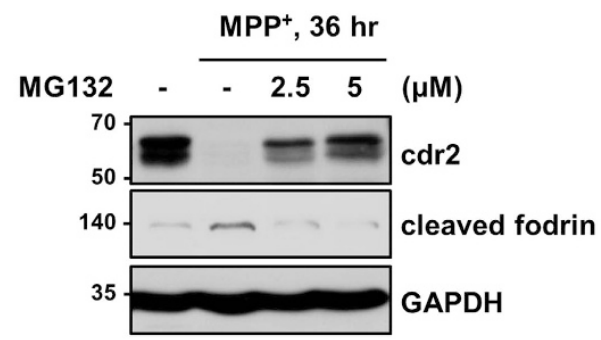

b

$\mathrm{MPP}^{+}, 36 \mathrm{hr}$

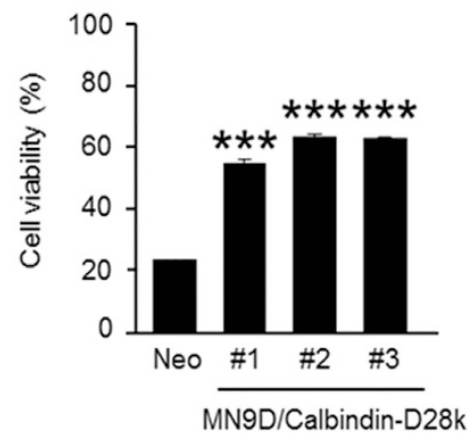

d
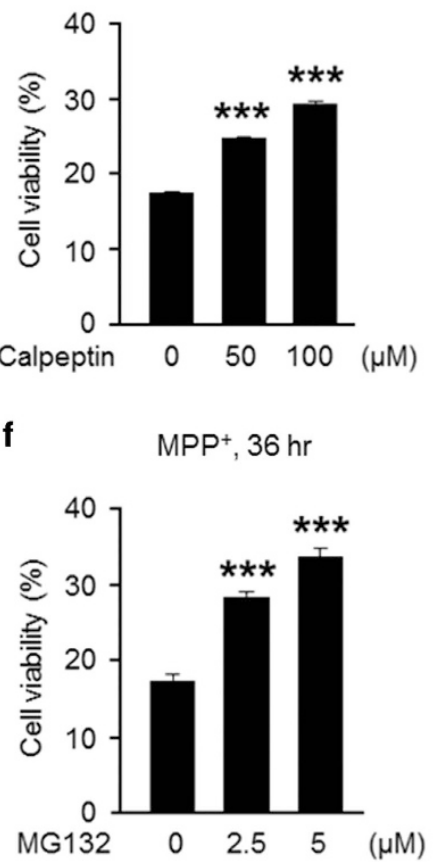

Figure 7 Preservation of cdr2 levels is linked to less vulnerability to MPP+-induced toxicity. (a) MN9D cells stably transfected with calbindin-D-28K (MN9D/calbindin-D-28K $\# 1$, \#2, or \#3) or control vector (MN9D/Neo) were exposed to $50 \mu \mathrm{M} \mathrm{MPP}^{+}$for $36 \mathrm{~h}$. Cell lysates from the indicated clones were processed for immunoblot analysis using the indicated antibodies. Mouse monoclonal anti-calbindin-D-28K antibody was used to detect its expression in stably established clones. (b) Cell viability was measured by MTT reduction assay. Data are shown as the mean \pm S.D. of three independent experiments. ${ }^{* * *} P<0.001$. (c-f) MN9D/Neo cells were treated with $50 \mu \mathrm{M}$ MPP ${ }^{+}$for $36 \mathrm{~h}$ in the presence or absence of the indicated concentration of calpeptin (c and $\mathbf{d}$ ) or MG132 (e and f). (c and e) Immunoblot analysis was performed using the indicated antibodies. (b, d, and f) Cell viability was expressed as a percentage of that for untreated control cells. Data are shown as the mean \pm S.D. of three independent experiments. ${ }^{\star \star \star} P<0.001$

Figure 6 Degradation of cdr2 by the ubiquitin proteasome system. (a) MN9D cells were treated with $50 \mu \mathrm{M} \mathrm{MPP}{ }^{+}$for $36 \mathrm{~h}$ in the presence or absence of calpeptin ( $\left.50 \mu \mathrm{M}\right)$, MG132 $(2.5 \mu \mathrm{M})$, clasto-lactacystin $\beta$-lactone $(2.5 \mu \mathrm{M})$, or Z-VAD-fmk $(100 \mu \mathrm{M})$. Levels of cdr2 were measured by immunoblot analysis using anti-cdr2 antibody. After normalization against GAPDH, levels of cdr2 were expressed as a percentage of that in untreated controls. Data are shown as the mean \pm S.D. of three independent experiments. ${ }^{*} P<0.05$; ${ }^{* *} P<0.01 ;{ }^{* \star *} P<0.001$; n.s., not significant. (b) ${ }^{35} S$-labeled cdr2 was incubated with or without a mixture of conjugation enzymes (E1, E2, and E3) plus ubiquitin and ubiquitin-aldehyde. Reaction mixtures were separated by SDS-PAGE and subjected to autoradiography. (c) MN9D cells transiently transfected with T7-tagged cdr2 plus HA-Ub were exposed to $50 \mu \mathrm{M} \mathrm{MPP}^{+}$for the indicated time period. To prevent proteasome-mediated degradation of cdr2, MG132 (2.5 $\left.\mu \mathrm{M}\right)$ was added to each culture $6 \mathrm{~h}$ before harvest. Cell lysates were processed for immunoprecipitation with mouse monoclonal anti-T7 and immunoblot analysis using mouse monoclonal anti-HA to detect poly-ubiquitinated cdr2. (d) To detect endogenous cdr2 ubiquitination, MN9D cells transiently transfected with HA-Ub were treated with or without $50 \mu \mathrm{M}$ MPP for $36 \mathrm{~h}$ in the presence of MG132 $(2.5 \mu \mathrm{M})$. Cell lysates were subjected to immunoprecipitation with anti-cdr2 antibody and followed by immunoblot analysis using mouse monoclonal anti-HA 
a
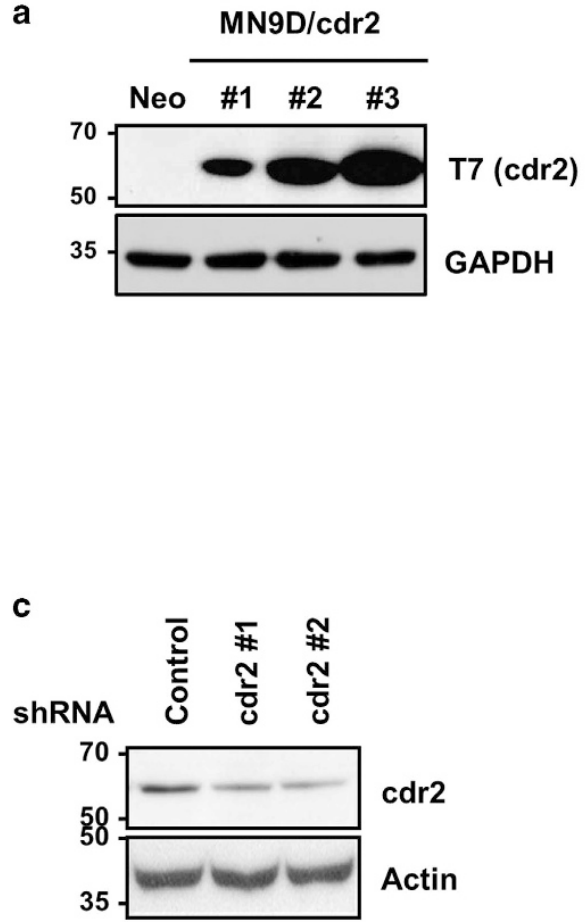

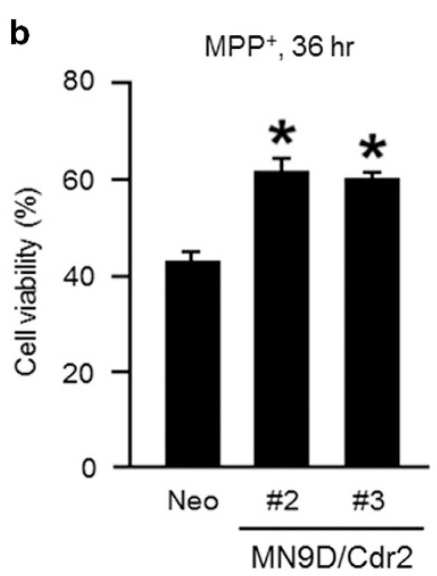

d

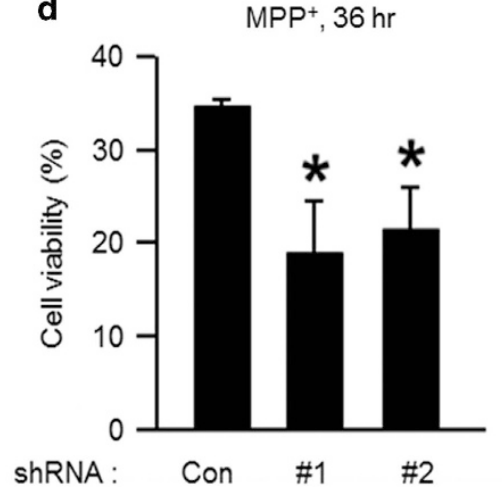

Figure 8 Protective role for cdr2 in MPP+-induced cell death. (a) MN9D cells were stably transfected with T7-tagged mouse cdr2 (MN9D/cdr2 \#1, \#2, or \#3) or empty vector (MN9D/Neo). Expression levels were validated by immunoblot analysis using mouse monoclonal anti-T7 antibody. (b) MN9D/Neo and two highly expressing MN9D/Cdr2 cell lines were treated with $50 \mu \mathrm{M} \mathrm{MPP}^{+}$for $36 \mathrm{~h}$. Cell viability was measured using MTT reduction assay and expressed as a percentage of that for untreated control cells. Data are shown as the mean \pm S.D. of three independent experiments. ${ }^{*} P<0.05$. (c) MN9D cells were stably transfected with cdr2 shRNA- or control shRNA-expressing vectors. Extent of cdr2 knockdown was determined by immunoblot analysis using anti-cdr2 antibody. (d) After treatment with $50 \mu \mathrm{M} \mathrm{MPP}{ }^{+}$for $36 \mathrm{~h}$, MTT reduction assay was performed. Cell viability was expressed as a percentage of that for untreated control cells. Data are shown as the mean \pm S.D. from three independent experiments. ${ }^{\star} P<0.05$

after surgery, $3-5$ rats in each condition were killed by $\mathrm{CO}_{2}$, and the $\mathrm{SN}$ was rapidly dissected out for immunological analysis. The SNs from four post-mortem patients with PD and four age-matched control individuals were provided by the Department of Pathology at Johns Hopkins University. Each brain underwent comprehensive neuropathological analysis. ${ }^{57}$

Primary neuronal cultures. To prepare primary cultures of dopaminergic neurons, the ventral mesencephalon was removed from SD rats (Orientbio) on embryonic day (E) 14 as previously described. ${ }^{28}$ Briefly, dopaminergic neuronal cultures were plated at $1.0 \times 10^{5}$ cells per $1 \mathrm{~cm}^{2}$ ACLAR embedding film (Electron Microscopy Sciences, Fort Washington, PA, USA) precoated with $100 \mu \mathrm{g} / \mathrm{ml}$ poly-D-lysine (Sigma) and $4 \mu \mathrm{g} / \mathrm{ml}$ laminin (Invitrogen, San Diego, CA, USA) and maintained at $37^{\circ} \mathrm{C}$ in a humidified $5 \% \mathrm{CO}_{2}$ atmosphere in modified Eagle's medium (MEM; Gibco, Grand Island, NY, USA) supplemented with $10 \%$ fetal bovine serum (FBS; Lonza, Walkersville, MD, USA), $2 \mathrm{mM}$ L-glutamine (Sigma), and $6 \mathrm{~g} / \mathrm{glucose}$ (Sigma). At 5 or 6 days in vitro (DIV), cultures were washed with MEM and treated with $3 \mu \mathrm{M} \mathrm{MPP}^{+}$for $36 \mathrm{~h}$.

MN9D cell culture, drug treatment, and cell viability. MN9D dopaminergic neuronal cultures were established from embryonic mesencephalic dopaminergic neurons by somatic fusion. ${ }^{35}$ As previously described by us, ${ }^{44}$ MN9D cells were plated on $25 \mu \mathrm{g} / \mathrm{ml}$ poly-D-lysine precoated culture dishes or plates (Costar, Corning, NY, USA), maintained in DMEM (Gibco) supplemented with $10 \%$ FBS in an incubator with $10 \% \mathrm{CO}_{2}$ at $37^{\circ} \mathrm{C}$, and switched to serum-free N2 medium ${ }^{58}$ containing various experimental reagents, including MPP $^{+}$, calpeptin (Calbiochem, San Diego, CA, USA), MG132 (Calbiochem), N-benzyloxycarbonylVal-Ala-Asp-fluoromethylketone (Z-VAD-fmk, Enzyme Systems Products, Livermore,
CA, USA), and clasto-lactacystin $\beta$-lactone (Calbiochem). To assess the rate of cell survival after drug treatment, MTT reduction assay was performed as previously described. ${ }^{59}$ Briefly, after the indicated incubation period, MTT solution was added to the culture at a final concentration of $1 \mathrm{mg} / \mathrm{ml}$. Cells were then incubated for $1 \mathrm{~h}$ at $37^{\circ} \mathrm{C}$ followed by lysis in $20 \%$ SDS in $50 \%$ aqueous dimethylformamide for $24 \mathrm{~h}$. The optical density of dissolved formazan grains was measured at $540 \mathrm{~nm}$ using a microplate reader (Molecular Devices, Sunnyvale, CA, USA). Values for each treatment group were calculated as a percentage relative to the untreated control group (defined as $100 \%$ survival).

Immunoblot analysis. The SN of post-mortem human brains was processed for immunoblot analysis as previously described by us. ${ }^{31}$ To measure cdr2 expression in rats, various body parts including brain regions were dissected and subjected to lysis. Briefly, dissected tissues were minced with blades, rinsed with ice-cold phosphate-buffered saline (PBS), and briefly microcentrifuged. The resulting pellets were lysed with RIPA buffer (50 mM Tris- $\mathrm{HCl}, \mathrm{pH} 7.4,1 \% \mathrm{NP}-40$, $0.25 \%$ sodium deoxycholate, $150 \mathrm{mM} \mathrm{NaCl}, 1 \mathrm{mM}$ EDTA) containing $0.1 \%$ SDS and protease inhibitor cocktail (Roche, Mannheim, Germany) and then further homogenized with a 1-ml syringe. SN obtained from rats that received stereotaxic injection of $\mathrm{MPP}^{+}$was similarly processed. For MN9D cells, cells were washed with ice-cold PBS, lysed with RIPA buffer containing protease inhibitor cocktail, and then homogenized using a 1-ml syringe. Tissue or cell lysates were microcentrifuged at $15000 \times g$ for $20 \mathrm{~min}$ at $4{ }^{\circ} \mathrm{C}$. Protein content of the supernatants was measured using a Bio-Rad protein assay reagent (Hercules, CA, USA). Proteins from each sample were separated on 10-12.5\% SDS-PAGE, blotted onto prewetted PVDF nitrocellulose filters (Bio-Rad), and blocked with TBST including 5\% skim milk for 
$1 \mathrm{~h}$. The blots were immunoprobed with human PCD patient serum that reacts with cdr2 (1:10000, generously provided by Dr Darnell at Rockefeller University, New York, NY, USA) or rabbit polyclonal anti-cdr2 (1:1000; Sigma), mouse monoclonal anti-tyrosine hydroxylase ( $\mathrm{TH}$, a rate-limiting enzyme of dopamine biosynthesis: 1: 1000; Pel-Freez, Rogers, AR, USA), mouse monoclonal anti- $\alpha$ fodrin (1: 4000; Enzo Life Science, Farmingdale, NY, USA), mouse monoclonal anti-hemagglutinin (HA; 1: 4000; Santa Cruz Biotechnology, Dallas, TX, USA), mouse monoclonal anti-T7 (1:1000; Novagen, Madison, WI, USA), mouse monoclonal anti-HSP 70 (1:1000; Santa Cruz), or mouse monoclonal anticalbindin-D-28K (1: 2000; Swant, Fribourg, Switzerland). Rabbit polyclonal antiactin (1: 4000; Sigma) and mouse monoclonal anti-glyceraldehyde-3 phosphate dehydrogenase (GAPDH, $1: 4000$; Merck Millipore, Billerica, MA, USA) were used as loading controls.

Immunofluorescent staining. For immunohistochemistry, rats were perfused transcardially with saline solution containing $0.5 \%$ sodium nitrate and heparin (1000 units $/ \mathrm{ml}$, Sigma) before fixation. Brains were fixed at $4{ }^{\circ} \mathrm{C}$ overnight with $4 \%$ paraformaldehyde in $0.1 \mathrm{M}$ phosphate buffer and incubated in $30 \%$ sucrose solution for $48-72 \mathrm{~h}$ at $4{ }^{\circ} \mathrm{C}$ until they sank. Brains were then cut into $30-\mu \mathrm{m}$-thick coronal sections using a sliding microtome. Sections were processed for immunohistochemical staining for $\mathrm{TH}$ and $\mathrm{cdr}$. For immunocytochemistry, primary cultures of mesencephalic cells and MN9D dopaminergic cells were fixed, blocked, and incubated with primary antibodies as previously described. ${ }^{28}$ Primary antibodies were mouse monoclonal anti-TH (1:7500, Pel-Freez), rabbit polyclonal GABA antibody (1:200; Sigma), and human PCD patient cerebrospinal fluid (CSF, $1: 20$; generously provided by Dr Darnell at Rockefeller University) that reacts with cdr2. After extensive washes with PBS, sections or cultures were incubated at RT for $1 \mathrm{~h}$ with Alexa 546-conjugated goat anti-mouse antibody or Alexa 546-conjugated goat anti-rabbit antibody in combination with Alexa 488-conjugated goat anti-human antibody (1: 200, Molecular Probes, Eugene, OR, USA). After extensive washes, sections or cultures were then mounted with Vectashield mounting medium (Vector Laboratories, Burlingame, CA, USA) and examined under a Axiovert 100 microscope equipped with an epifluorescence and digital image analyzer (Carl Zeiss, Zena, Germany) or an LSM 510 Meta Laser Scanning Microscope (Carl Zeiss). Fluorescence intensity was measured and analyzed using ImageJ software $(\mathrm{NIH}$, Bethesda, MD, USA).

Measurement of cytosolic free $\mathrm{Ca}^{2+}$ by Fluo-3. A method for visualization of intracellular free $\mathrm{Ca}^{2+}$ levels was applied to MN9D cells using Fluo-3 calcium indicator (Molecular Probes). Briefly, MN9D cells treated with or without $50 \mu \mathrm{M} \mathrm{MPP}^{+}$for the indicated time were loaded with $4 \mu \mathrm{M}$ Fluo-3 AM and incubated at $37^{\circ} \mathrm{C}$ for $30 \mathrm{~min}$. After incubation, cells were washed twice with N2-supplemented medium and examined under an Axiovert 100 microscope equipped with an epifluorescence and digital image analyzer (Carl Zeiss) at an excitation wavelength of $488 \mathrm{~nm}$.

In vitro and cell-based calpain cleavage assay. For in vitro calpain cleavage assays, the vector encoding T7-tagged mouse cdr2 in pcDNA3 was transcribed and translated in the presence of ${ }^{35} \mathrm{~S}$-methionine (Perkin Elmer, Boston, MA, USA) using a TnT Quick coupled transcription/translation system (Promega, Madison, WI, USA) according to the manufacturer's recommendations. For cell-based cleavage assay, MN9D cells were lysed in buffer containing $50 \mathrm{mM}$ Tris- $\mathrm{HCl}, \mathrm{pH}$ 8.0, $2 \mathrm{mM}$ EDTA, and 1\% Triton X-100 buffer without protease inhibitor cocktail. $\left[{ }^{35} \mathrm{~S}\right]-c d r 2$ or cell lysates $(50 \mu \mathrm{g})$ were incubated for $1 \mathrm{~h}$ at $30{ }^{\circ} \mathrm{C}$ in a calpain activation buffer containing $1 \mathrm{mM} \mathrm{CaCl} 2$ in the presence or absence of purified m-calpain ( 0.343 units) or $\mu$-calpain $(0.134$ units; both from Calbiochem) as recommended by the manufacturer. If necessary, calpeptin $(50 \mu \mathrm{M})$ or MG132 $(2.5 \mu \mathrm{M})$ was added to the reaction mixtures. Reactions were terminated by the addition of $5 x$ protein sample buffer followed by boiling for $5 \mathrm{~min}$. The resulting products were separated on 10\% SDS-PAGE gel and processed for autoradiography or immunoblot analysis.

In vitro and cell-based ubiquitination assay. By using an ubiquitin protein conjugating kit (Calbiochem), in vitro ubiquitination of $\left.{ }^{35} \mathrm{~S}\right]$-cdr2 was performed as recommended by the manufacturer. Briefly, all components mixed at a total volume of $25 \mu \mathrm{l}$ were incubated for $3 \mathrm{~h}$ at $37^{\circ} \mathrm{C}$. The reaction was terminated by adding $5 \times$ protein sample buffer and boiling for $5 \mathrm{~min}$. The reaction mixtures were separated on $8 \%$ SDS-PAGE gel and processed for autoradiography. To assess the ubiquitination pattern of exogenous and endogenous cdr2, MN9D cells were transiently transfected with or without T7-tagged cdr2 in combination with $\mathrm{HA}-\mathrm{Ub}$ and exposed to $50 \mu \mathrm{M} \mathrm{MPP}{ }^{+}$for the indicated period of time. To inhibit proteasome-mediated degradation of the ubiquitinated proteins, $2.5 \mu \mathrm{M}$ MG132 was added to the culture medium. Cells were then harvested and subjected to lysis in RIPA buffer containing protease inhibitor cocktail. For immunoprecipitation, lysates $(500 \mu \mathrm{g})$ were precleared with protein A agarose (Upstate Biotechnology, Lake Placid, NY, USA) for $2 \mathrm{~h}$ and further incubated with either mouse monoclonal antiT7 antibody or rabbit polyclonal anti-cdr2 antibody (Sigma) with gentle rotation overnight at $4{ }^{\circ} \mathrm{C}$. Immunocomplexes were collected by incubation with protein $\mathrm{A}$ agarose for $2 \mathrm{~h}$ at $4{ }^{\circ} \mathrm{C}$ and subjected to centrifugation at $3000 \times g$ at $4{ }^{\circ} \mathrm{C}$ for $2 \mathrm{~min}$. After washing the beads three times, proteins were eluted by boiling with $1 \times$ protein sample buffer and then separated on SDS-PAGE gel and subjected to immunoblot analysis using mouse monoclonal anti-ubiquitin antibody (Santa Cruz).

Plasmids and transfection. T7-tagged full-length mouse cdr2 (GenBank Accession Number U88588) in pcDNA3 eukaryotic expression vector was generously provided from Dr Darnell at Rockefeller University. For constructs expressing cdr2specific shRNA, the sequences $5^{\prime}$-GGATCCCGTTGATGCAACTAAATATCTCCTTGAT ATCCGGGAGATATTTAGTTGCATCAATTTTTTCCAAAAGCTT-3' (\#1) or 5'-GGATCC CGTTTCGCATGCTGCTCATTCATTTGATATCCGATGAATGAGCAGCATGCGAAATTT TTTCCAAAAGCTT-3' (\#2) were chosen based on recommendations by Genscript's shRNA design center (http://www.genscript.com/design_center.html) and ligated into pRNAT-U6.1/Neo vectors. GFP signal under control of the CMV promoter in the vectors was used to track transfection efficiency. MN9D cells were transfected with vector containing T7-tagged cdr2 or cdr2 shRNAs using Lipofectamine 2000 (Invitrogen) as recommended by the manufacturer. The transfected cells were maintained in the presence of $500 \mu \mathrm{g} / \mathrm{ml}$ G418 (AG Scientific Inc., San Diego, CA, USA) for an additional 2 weeks and expanded in culture medium containing $250 \mu \mathrm{g} / \mathrm{ml} \mathrm{G} 418$ for further experiments.

Statistical analyses. Data are shown as mean \pm S.D. Group differences were analyzed using Student's $t$-tests (unpaired, two tailed) or one-way analysis of variance (ANOVA) followed by Tukey's post hoc tests using GraphPad Prism 6 software (La Jolla, CA, USA). Statistical significance was set at $P<0.05$.

\section{Conflict of Interest}

The authors declare no conflict of interest.

Acknowledgements. This research was supported by the Mid-Career Research Program through the National Research Foundation (NRF) funded by the Ministry of Education, Science, and Technology (to YJO), and by the Brain Research Program through NRF funded by the Ministry of Science, ICT \& Future Planning: 2014M3C7A1064545 to KCC and 2012M2A2A7010422 to H-DU. JL is a recipient of the Brain Research Program through the NRF funded by the Ministry of Science, ICT, and Future Planning (2013M3C7A1056731).

1. Albert ML, Darnell RB. Paraneoplastic neurological degenerations: keys to tumour immunity. Nat Rev Cancer 2004; 4: 36-44.

2. Darnell RB, Posner JB. Paraneoplastic syndromes affecting the nervous system. Semin Oncol 2006; 33: 270-298.

3. Storstein A, Vedeler CA. Paraneoplastic neurological syndromes and onconeural antibodies: clinical and immunological aspects. Adv Clin Chem 2007; 44: 143-185.

4. Corradi JP, Yang C, Darnell JC, Dalmau J, Darnell RB. A post-transcriptional regulatory mechanism restricts expression of the paraneoplastic cerebellar degeneration antigen cdr2 to immune privileged tissues. J Neurosci 1997; 17: 1406-1415.

5. Roberts WK, Darnell RB. Neuroimmunology of the paraneoplastic neurological degenerations. Curr Opin Immunol 2004; 16: 616-622.

6. Greenlee JE, Brashear HR. Antibodies to cerebellar Purkinje cells in patients with paraneoplastic cerebellar degeneration and ovarian carcinoma. Ann Neurol 1983; 14: 609-613.

7. Cunningham J, Graus F, Anderson N, Posner JB. Partial characterization of the Purkinje cell antigens in paraneoplastic cerebellar degeneration. Neurology 1986; 36: 1163-1168.

8. Peterson K, Rosenblum MK, Kotanides H, Posner JB. Paraneoplastic cerebellar degeneration. I. A clinical analysis of 55 anti-Yo antibody-positive patients. Neurology 1992: 42: 1931-1937.

9. Darnell RB. Onconeural antigens and the paraneoplastic neurologic disorders: at the intersection of cancer, immunity, and the brain. Proc Natl Acad Sci USA 1996; 93: 4529-4536.

10. Okano HJ, Park WY, Corradi JP, Darnell RB. The cytoplasmic Purkinje onconeural antigen cdr2 down-regulates c-Myc function: implications for neuronal and tumor cell survival. Genes Dev 1999; 13: 2087-2097 
11. Darnell RB, Posner JB. Paraneoplastic syndromes involving the nervous system. $N$ Eng $J$ Med 2003; 349: 1543-1554.

12. Greenlee JE, Clawson SA, Hill KE, Wood BL, Tsunoda I, Carlson NG. Purkinje cell death after uptake of anti-Yo antibodies in cerebellar slice cultures. J Neuropathol Exp Neurol 2010; 69: 997-1007.

13. Takanaga $H$, Mukai $H$, Shibata $H$, Toshimori M, Ono Y. PKN interacts with a paraneoplastic cerebellar degeneration-associated antigen, which is a potential transcription factor. Exp Cell Res 1998; 241: 363-372.

14. O'Donovan KJ, Diedler J, Couture GC, Fak JJ, Darnell RB. The onconeural antigen cdr2 is a novel $\mathrm{APC} / \mathrm{C}$ target that acts in mitosis to regulate $\mathrm{c}$-myc target genes in mammalian tumor cells. PloS One 2010; 5: e10045.

15. Jankovic J. Parkinson's disease: clinical features and diagnosis. I Neurol Neurosurg Psychiatry 2008; 79: 368-376.

16. Dauer W, Przedborski S. Parkinson's disease: mechanisms and models. Neuron 2003; 39 : 889-909.

17. Moore DJ, West AB, Dawson VL, Dawson TM. Molecular pathophysiology of Parkinson's disease. Annu Rev Neurosci 2005; 28: 57-87.

18. Hartmann A, Hunot S, Michel PP, Muriel MP, Vyas S, Faucheux BA et al. Caspase-3: a vulnerability factor and final effector in apoptotic death of dopaminergic neurons in Parkinson's disease. Proc Natl Acad Sci USA 2000; 97: 2875-2880.

19. Viswanath V, Wu Y, Boonplueang R, Chen S, Stevenson FF, Yantirin F et al. Caspase- 9 activation results in downstream caspase-8 activation and bid cleavage in 1-methyl-4-phenyl1,2,3,6-tetrahydropyridine-induced Parkinson's disease. J Neurosci 2001; 21: 9519-9528.

20. Bilsland J, Roy S, Xanthoudakis S, Nicholson DW, Han Y, Grimm E et al. Caspase inhibitors attenuate 1-methyl-4-phenylpyridinium toxicity in primary cultures of mesencephalic dopaminergic neurons. J Neurosci 2002; 22: 2637-2649.

21. Mishizen-Eberz AJ, Guttmann RP, Giasson BI, Day GA 3rd, Hodara R, Ischiropoulos $\mathrm{H}$ et al. Distinct cleavage patterns of normal and pathologic forms of alpha-synuclein by calpain I in vitro. J Neurochem 2003; 86: 836-847.

22. Smith PD, Mount MP, Shree R, Callaghan S, Slack RS, Anisman H et al. Calpain-regulated p35/cdk5 plays a central role in dopaminergic neuron death through modulation of the transcription factor myocyte enhancer factor 2. J Neurosci 2006; 26: 440-447.

23. Samantaray S, Ray SK, Banik NL. Calpain as a potential therapeutic target in Parkinson's disease. CNS Neurol Disord Drug Targets 2008; 7: 305-312.

24. Camins A, Crespo-Biel N, Junyent F, Verdaguer E, Canudas AM, Pallas M. Calpains as a target for therapy of neurodegenerative diseases: putative role of lithium. Curr Drug Metab 2009; 10: 433-447.

25. Harbison RA, Ryan KR, Wilkins HM, Schroeder EK, Loucks FA, Bouchard RJ et al. Calpain plays a central role in 1-methyl-4-phenylpyridinium (MPP+)-induced neurotoxicity in cerebellar granule neurons. Neurotox Res 2011; 19: 374-388.

26. Diepenbroek M, Casadei N, Esmer H, Saido TC, Takano J, Kahle PJ et al. Overexpression of the calpain-specific inhibitor calpastatin reduces human alpha-Synuclein processing, aggregation and synaptic impairment in [A30P]alphaSyn transgenic mice. Hum Mol Genet 2014; 23: 3975-3989.

27. Samantaray S, Knaryan VH, Shields DC, Cox A, Haque A, Banik NL. Inhibition of calpain activation protects MPTP-induced nigral and spinal cord neurodegeneration, reduces inflammation, and improves gait dynamics in mice. Mol Neurobiol 2015; 52: 1054-1066.

28. Han BS, Hong HS, Choi WS, Markelonis GJ, Oh TH, Oh YJ. Caspase-dependent and -independent cell death pathways in primary cultures of mesencephalic dopaminergic neurons after neurotoxin treatment. J Neurosci 2003; 23: 5069-5078.

29. Choi WS, Eom DS, Han BS, Kim WK, Han BH, Choi EJ et al. Phosphorylation of p38 MAPK induced by oxidative stress is linked to activation of both caspase-8- and -9-mediated apoptotic pathways in dopaminergic neurons. J Biol Chem 2004; 279: 20451-20460.

30. Kim C, Yun N, Lee YM, Jeong JY, Baek JY, Song H et al. Gel-based protease proteomics for identifying the novel calpain substrates in dopaminergic neuronal cell. J Biol Chem 2013; 288: 36717-36732

31. Yun N, Lee YM, Kim C, Shibayama H, Tanimura A, Hamanaka Y et al. Anamorsin, a nove caspase-3 substrate in neurodegeneration. J Biol Chem 2014; 289: 22183-22195.

32. Darnell JC, Albert ML, Darnell RB. Cdr2, a target antigen of naturally occuring human tumor immunity, is widely expressed in gynecological tumors. Cancer Res 2000; 60: 2136-2139.

33. Kawamata T, Taniguchi T, Mukai H, Kitagawa M, Hashimoto T, Maeda K et al. A protein kinase, PKN, accumulates in Alzheimer neurofibrillary tangles and associated endoplasmic reticulum-derived vesicles and phosphorylates tau protein. J Neurosci 1998; 18: 7402-7410.

34. Manser C, Stevenson A, Banner S, Davies J, Tudor EL, Ono Y et al. Deregulation of PKN1 activity disrupts neurofilament organisation and axonal transport. FEBS Lett 2008; 582: 2303-2308.

35. Choi HK, Won LA, Kontur PJ, Hammond DN, Fox AP, Wainer BH et al. Immortalization of embryonic mesencephalic dopaminergic neurons by somatic cell fusion. Brain Res 1991; 552: 67-76.

36. Tang L, Todd RD, Heller A, O'Malley KL. Pharmacological and functional characterization of D2, D3 and D4 dopamine receptors in fibroblast and dopaminergic cell lines. J Pharmacol Exp Ther 1994; 268: 495-502.

37. Choi WS, Lee E, Lim J, Oh YJ. Calbindin-D28K prevents drug-induced dopaminergic neuronal death by inhibiting caspase and calpain activity. Biochem Biophys Res Commun 2008; 371: 127-131.
38. Lim J, Lee Y, Jung S, Youdim MB, Oh YJ. Impaired autophagic flux is critically involved in drug-induced dopaminergic neuronal death. Parkinsonism Relat Disord 2014; 20(Suppl 1): S162-S166.

39. Tsubuki S, Saito Y, Tomioka M, Ito H, Kawashima S. Differential inhibition of calpain and proteasome activities by peptidyl aldehydes of di-leucine and tri-leucine. J Biol Chem 1996; 119: $572-576$.

40. Persechini A, Moncrief ND, Kretsinger RH. The EF-hand family of calcium-modulated proteins. Trends Neurosci 1989; 12: 462-467.

41. Baimbridge KG, Celio MR, Rogers $\mathrm{JH}$. Calcium-binding proteins in the nervous system. Trends Neurosci 1992; 15: 303-308.

42. Choi WS, Kruse SE, Palmiter RD, Xia Z. Mitochondrial complex I inhibition is not required for dopaminergic neuron death induced by rotenone, MPP+, or paraquat. Proc Natl Acad Sci USA 2008; 105: 15136-15141.

43. Kubbutat $\mathrm{MH}$, Vousden $\mathrm{KH}$. Proteolytic cleavage of human p53 by calpain: a potential regulator of protein stability. Mol Cell Biol 1997; 17: 460-468.

44. Choi WS, Lee EH, Chung CW, Jung YK, Jin BK, Kim SU et al. Cleavage of Bax is mediated by caspase-dependent or -independent calpain activation in dopaminergic neuronal cells: protective role of Bcl-2. J Neurochem 2001; 77: 1531-1541.

45. Banik NL, Shields DC, Ray S, Davis B, Matzelle D, Wilford G et al. Role of calpain in spinal cord injury: effects of calpain and free radical inhibitors. Ann NY Acad Sci 1998; 844: 131-137.

46. Kristian T, Siesjo BK. Calcium in ischemic cell death. Stroke 1998; 29,:705-718.

47. Ray SK, Hogan EL, Banik NL. Calpain in the pathophysiology of spinal cord injury: neuroprotection with calpain inhibitors. Brain Res Brain Res Rev 2003; 42: 169-185.

48. Peng S, Kuang Z, Zhang Y, Xu H, Cheng Q. The protective effects and potential mechanism of Calpain inhibitor Calpeptin against focal cerebral ischemia-reperfusion injury in rats. $\mathrm{Mol}$ Biol Rep 2011; 38: 905-912.

49. Dekkers MP, Nikoletopoulou V, Barde YA. Cell biology in neuroscience: death of developing neurons: new insights and implications for connectivity. J Cell Biol 2013; 203: 385-393.

50. Fathallah-Shaykh H, Wolf S, Wong E, Posner JB, Furneaux HM. Cloning of a leucine-zipper protein recognized by the sera of patients with antibody-associated paraneoplastic cerebellar degeneration. Proc Natl Acad Sci USA 1991; 88: 3451-3454.

51. Hida C, Tsukamoto T, Awano H, Yamamoto T. Ultrastructural localization of anti-Purkinje cell antibody-binding sites in paraneoplastic cerebellar degeneration. Arch Neurol 1994; 51: 555-558.

52. Sakai K, Shirakawa T, Li Y, Kitagawa Y, Hirose G. Interaction of a paraneoplastic cerebellar degeneration-associated neuronal protein with the nuclear helix-loop-helix leucine zipper protein MRG X. Mol Cell Neurosci 2002; 19: 477-484.

53. Sakai K, Kitagawa Y, Saiki S, Saiki M, Hirose G. Effect of a paraneoplastic cerebellar degeneration-associated neural protein on B-myb promoter activity. Neurobiol Dis 2004; 15 : 529-533.

54. Liu DX, Biswas SC, Greene LA. B-myb and C-myb play required roles in neuronal apoptosis evoked by nerve growth factor deprivation and DNA damage. J Neurosci 2004; 24 : $8720-8725$.

55. Park ES, Kim SR, Jin BK. Transient receptor potential vanilloid subtype 1 contributes to mesencephalic dopaminergic neuronal survival by inhibiting microglia-originated oxidative stress. Brain Res Bull 2012; 89: 92-96.

56. Paxinos G, Watson C (eds). The Rat Brain: In stereotaxic coordinates, 4th edn. Elsevier/ Academic Press: NY, USA, 1998.

57. Shin JH, Ko HS, Kang H, Lee Y, Lee YI, Pletinkova O et al. PARIS (ZNF746) repression of PGC-1alpha contributes to neurodegeneration in Parkinson's disease. Cell 2011; 144: 689-702.

58. Bottenstein JE, Sato GH. Growth of a rat neuroblastoma cell line in serum-free supplemented medium. Proc Natl Acad Sci USA 1979; 76: 514-517.

59. Shearman MS, Ragan $\mathrm{Cl}$, Iversen LL. Inhibition of $\mathrm{PC} 12$ cell redox activity is a specific, early indicator of the mechanism of beta-amyloid-mediated cell death. Proc Natl Acad Sci USA 1994; 91: 1470-1474.

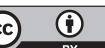

Cell Death and Disease is an open-access journal published by Nature Publishing Group. This work is licensed under a Creative Commons Attribution 4.0 International License. The images or other third party material in this article are included in the article's Creative Commons license, unless indicated otherwise in the credit line; if the material is not included under the Creative Commons license, users will need to obtain permission from the license holder to reproduce the material. To view a copy of this license, visit http://creativecommons.org/licenses/by/4.0/ 